\title{
Alboatisins A-C, ent-Atisene Diterpenoids from Isodon albopilosus
}

Sheng-Xiong Huang, Yan Zhou, Li-Bin Yang, Yong Zhao, Sheng-Hong Li, Li-Guang Lou, Quan-Bin Han, Li-Sheng Ding, and Han-Dong Sun

\section{Supporting Information Available}

\section{Contents:}

1. ${ }^{1} \mathrm{H}$ and ${ }^{13} \mathrm{C}$ NMR data of alboatisin A (1) and rabdokunmin C 2

2. Proposed biogenetic pathway for $\mathbf{1} 2$

3. EIMS of alboatisin A (1) 3

4. HRESIMS of alboatisin A (1) 4

5. ${ }^{1} \mathrm{H}$ NMR spectrum of alboatisin A (1) 5

6. ${ }^{13} \mathrm{C}$ NMR spectrum of alboatisin A (1) 6

7. $\quad$ DEPT NMR spectra of alboatisin A (1) 7

8. HSQC NMR spectrum of alboatisin A (1) 8

9. COSY NMR spectrum of alboatisin A (1) 9

10. HMBC NMR spectrum of alboatisin A (1) 10

11. Expanded HMBC spectrum of alboatisin A (1) 11

12. ROESY NMR spectrum of alboatisin A (1) 12

13. ${ }^{1} \mathrm{H}$ NMR spectrum of alboatisin B (2) 13

14. ${ }^{13}$ C NMR spectrum of alboatisin B (2) 14

15. HMBC NMR spectrum of alboatisin B (2) 15

16. ${ }^{1} \mathrm{H}$ NMR spectrum of alboatisin C (3) 16

17. ${ }^{13} \mathrm{C}$ NMR spectrum of alboatisin C (3) 17

18. $\quad$ COSY NMR spectrum of alboatisin C (3) 18 


\section{${ }^{1} \mathrm{H}$ and ${ }^{13} \mathrm{C}$ NMR data of alboatisin A (1) and rabdokunmin C}

\begin{tabular}{|c|c|c|c|c|}
\hline & \multicolumn{2}{|l|}{ alboatisin A (1) } & \multicolumn{2}{|c|}{ rabdokunmin $\mathrm{C}$} \\
\hline & ${ }^{1} \mathrm{H}$ (mult., $J$ in $\mathrm{Hz}$ ) & ${ }^{13} \mathrm{C}$ (mult.) & ${ }^{1} \mathrm{H}$ (mult., $J$ in $\mathrm{Hz}$ ) & ${ }^{13} \mathrm{C}$ (mult.) \\
\hline 1 & $1.54(\mathrm{~m})$ and $0.72(\mathrm{~m})$ & $39.0(\mathrm{t})$ & $1.95(\mathrm{~m})$ and $0.86(\mathrm{~m})$ & $39.5(\mathrm{t})$ \\
\hline 2 & $1.53(\mathrm{~m})$ and $1.62(\mathrm{~m})$ & $18.2(\mathrm{t})$ & $1.42-1.70(2 \mathrm{H}, \mathrm{m})$ & $18.3(\mathrm{t})$ \\
\hline 3 & $1.75(\mathrm{~m})$ and $1.38(\mathrm{~m})$ & $35.7(t)$ & $1.86(\mathrm{~m}) 1.38(\mathrm{~m})$ & $35.6(\mathrm{t})$ \\
\hline 4 & & $37.8(\mathrm{~s})$ & & $38.7(\mathrm{~s})$ \\
\hline 5 & $1.62(\mathrm{~m})$ & 46.0 (d) & $1.75(\mathrm{~m})$ & 46.5 (d) \\
\hline 6 & $2.36(\mathrm{dd}, 12.8,4.1)$ and $2.01(\mathrm{~m})$ & $29.0(\mathrm{t})$ & $2.44(\mathrm{~m})$ and $2.00(\mathrm{~m})$ & $29.7(\mathrm{t})$ \\
\hline 7 & $5.05\left(\right.$ in $\left.\mathrm{H}_{2} \mathrm{O}\right)$ & 71.9 (d) & $4.88\left(\right.$ in $\left.\mathrm{H}_{2} \mathrm{O}\right)$ & 74.5 (d) \\
\hline 8 & & $56.6(\mathrm{~s})$ & & $61.7(\mathrm{~s})$ \\
\hline 9 & $1.46(\mathrm{~m})$ & 48.0 (d) & $2.05(\mathrm{~m})$ & 57.2 (d) \\
\hline 10 & & $39.0(\mathrm{~s})$ & & $38.0(\mathrm{~s})$ \\
\hline 11 & $2.41(\mathrm{dd}, 12.4,7.7)$ and $1.61(\mathrm{~m})$ & $20.7(t)$ & $2.03(\mathrm{~m})$ and $1.88(\mathrm{~m})$ & $26.5(t)$ \\
\hline 12 & 3.17 (brs) & 43.9 (d) & $4.35(\mathrm{~m})$ & 72.5 (d) \\
\hline 13 & 4.48 (brs) & $76.8(d)$ & $3.58(\mathrm{~m})$ & $55.6(d)$ \\
\hline 14 & 5.11 (brs) & 75.7 (d) & 5.82 (brs) & 71.3 (d) \\
\hline 15 & & $200.8(s)$ & & $209.0(\mathrm{~s})$ \\
\hline 16 & & $145.6(\mathrm{~s})$ & & $147.7(\mathrm{~s})$ \\
\hline 17 & 6.26 (brs) and 5.30 (brs) & $118.0(\mathrm{t})$ & 6.29 (brs) and 5.42 (brs) & $117.0(\mathrm{t})$ \\
\hline 18 & 3.65 and $3.32(\mathrm{~d}, 10.8)$ & $71.3(\mathrm{t})$ & 3.66 and $3.32(\mathrm{~d}, 10.8)$ & $71.3(\mathrm{t})$ \\
\hline 19 & $0.86(\mathrm{~s})$ & $18.1(q)$ & $0.87(\mathrm{~s})$ & $18.0(q)$ \\
\hline 20 & $1.40(\mathrm{~s})$ & $16.6(\mathrm{q})$ & $1.67(\mathrm{~s})$ & 17.0 (q) \\
\hline
\end{tabular}

\section{Proposed biogenetic pathway for 1:}

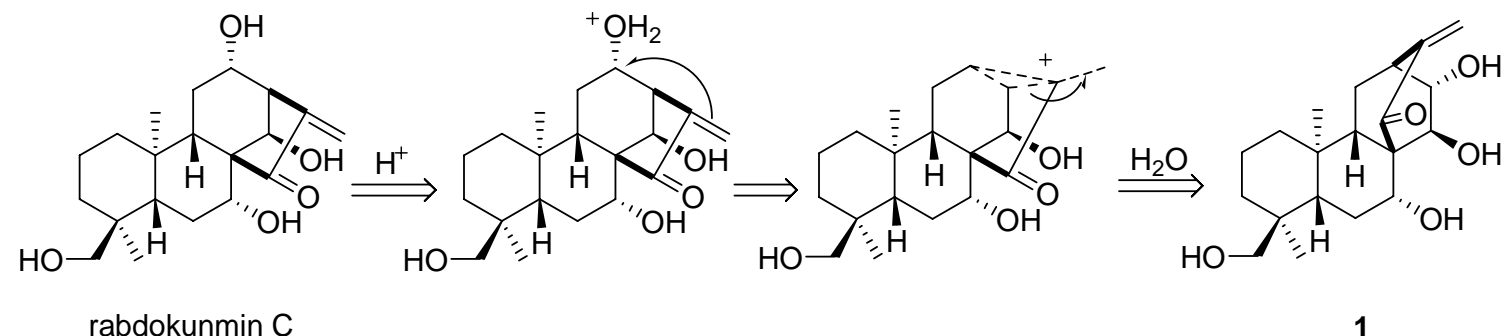

rabdokunmin C 


\section{EIMS of alboatisin A (1)}

C: Ucaliburldatal0118e-09

01/18/2005 04:23:28 PM

0118e-09 \#205-212 RT: $2.21-2.27$ AV: 8 SB: 16 3.05-3.17, 3.17 NL: 6.65E6

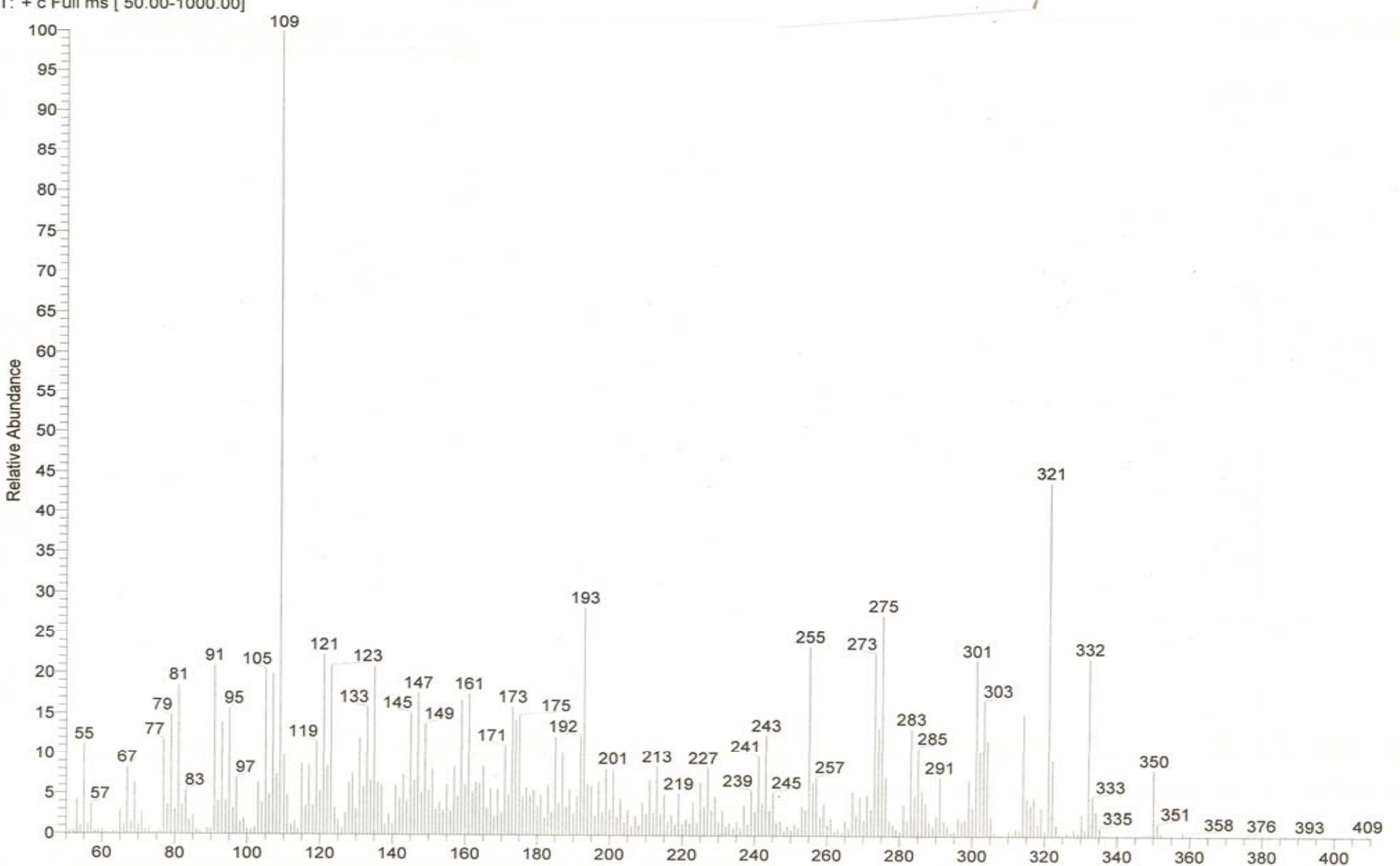


HRESIMS of alboatisin A (1)

Acq. Date: Wednesday, January 19, 2005

Acq. Time: $09: 33$

Scan Mode: zero width

Sample Name: 050119ESIA shsx-26 Sample comment

+TOF MS: 3.050 to 3.100 min from 050119ESIA shsx-26. wiff

Max. 1066.3 counts.

$\mathrm{a}=3.55849423347743840 \mathrm{e}-004, \mathrm{t} 0=7.96575916585934460 \mathrm{e}+001$

373.1984

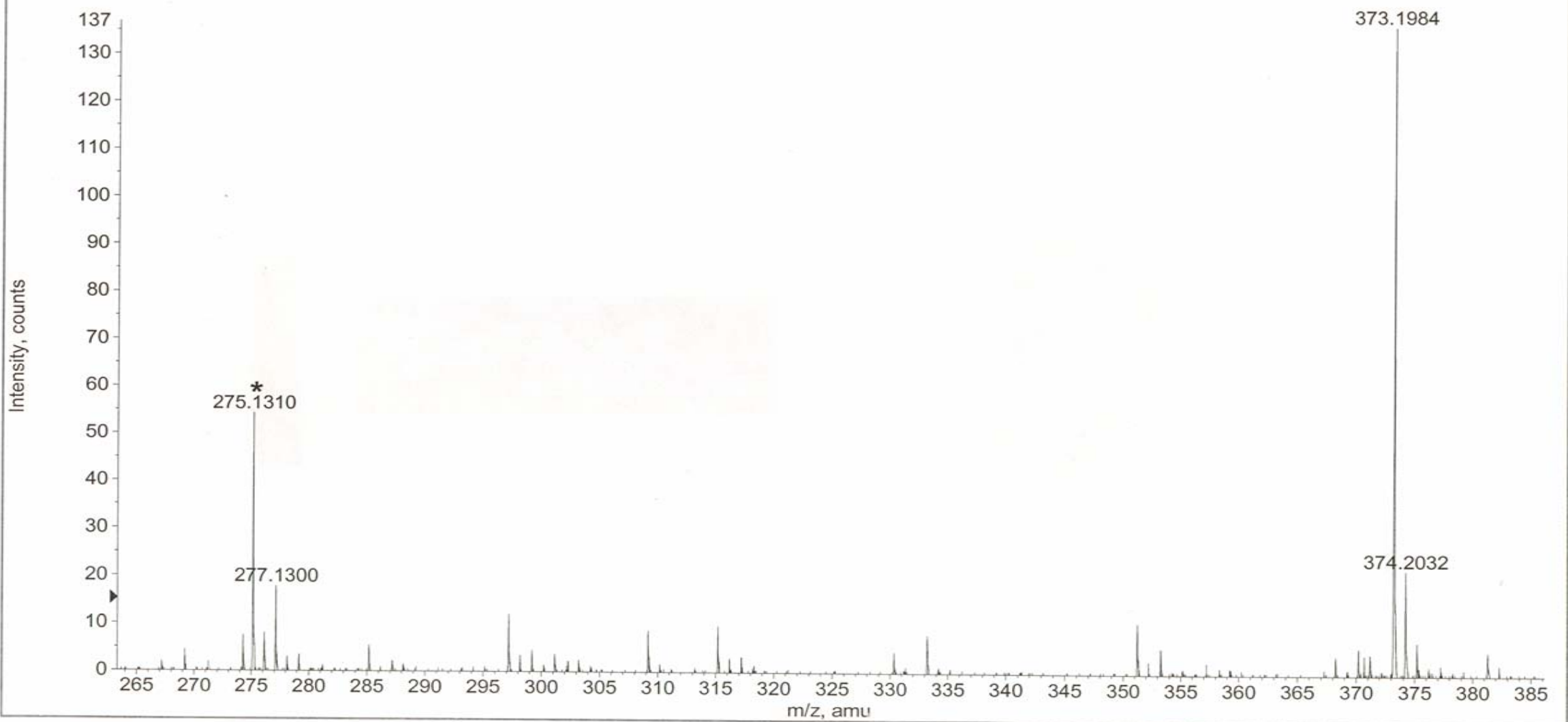




\section{${ }^{1} \mathrm{H}$ NMR spectrum of alboatisin A (1)}

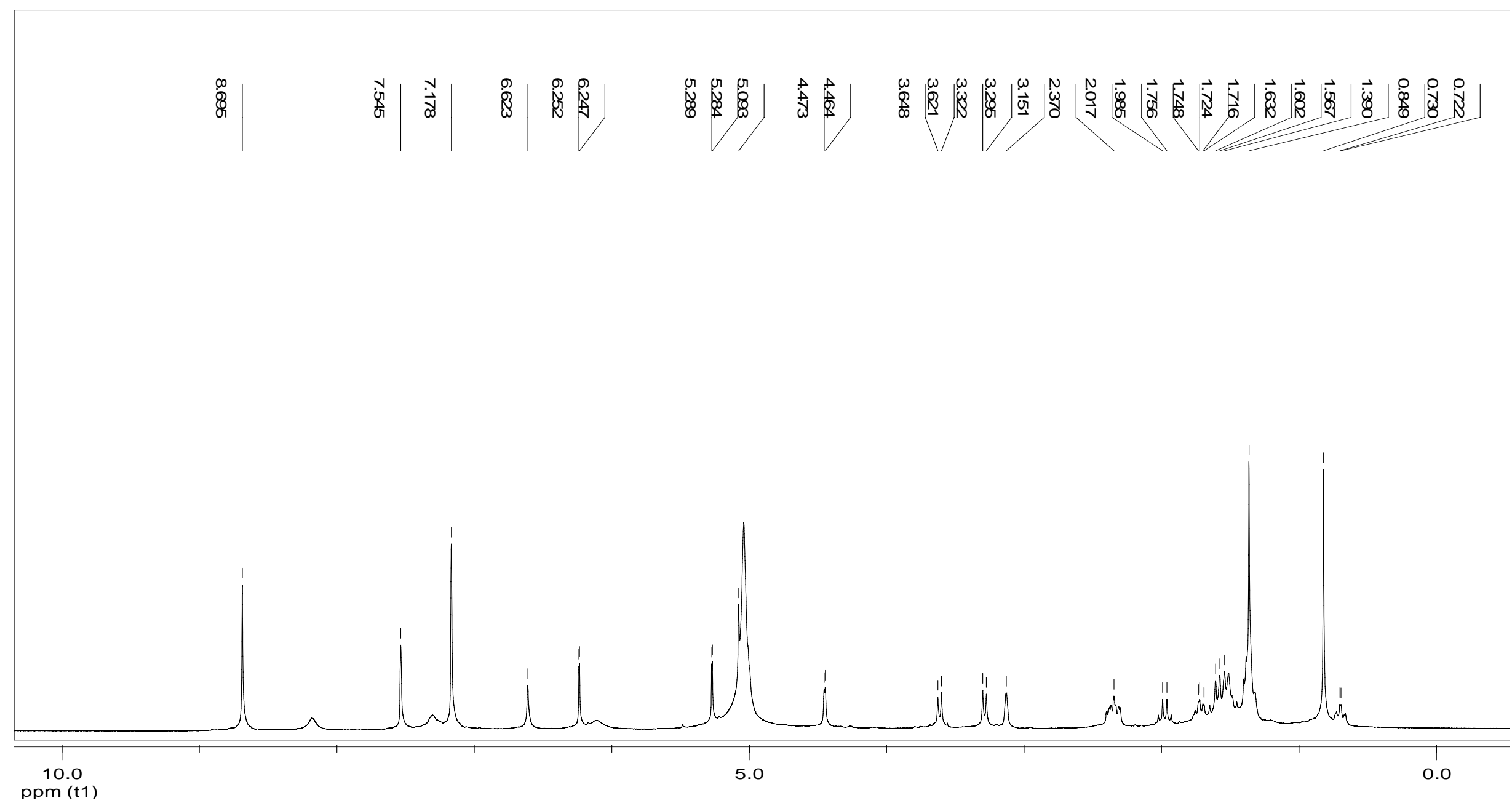


${ }^{13} \mathrm{C}$ NMR spectrum of alboatisin A (1)

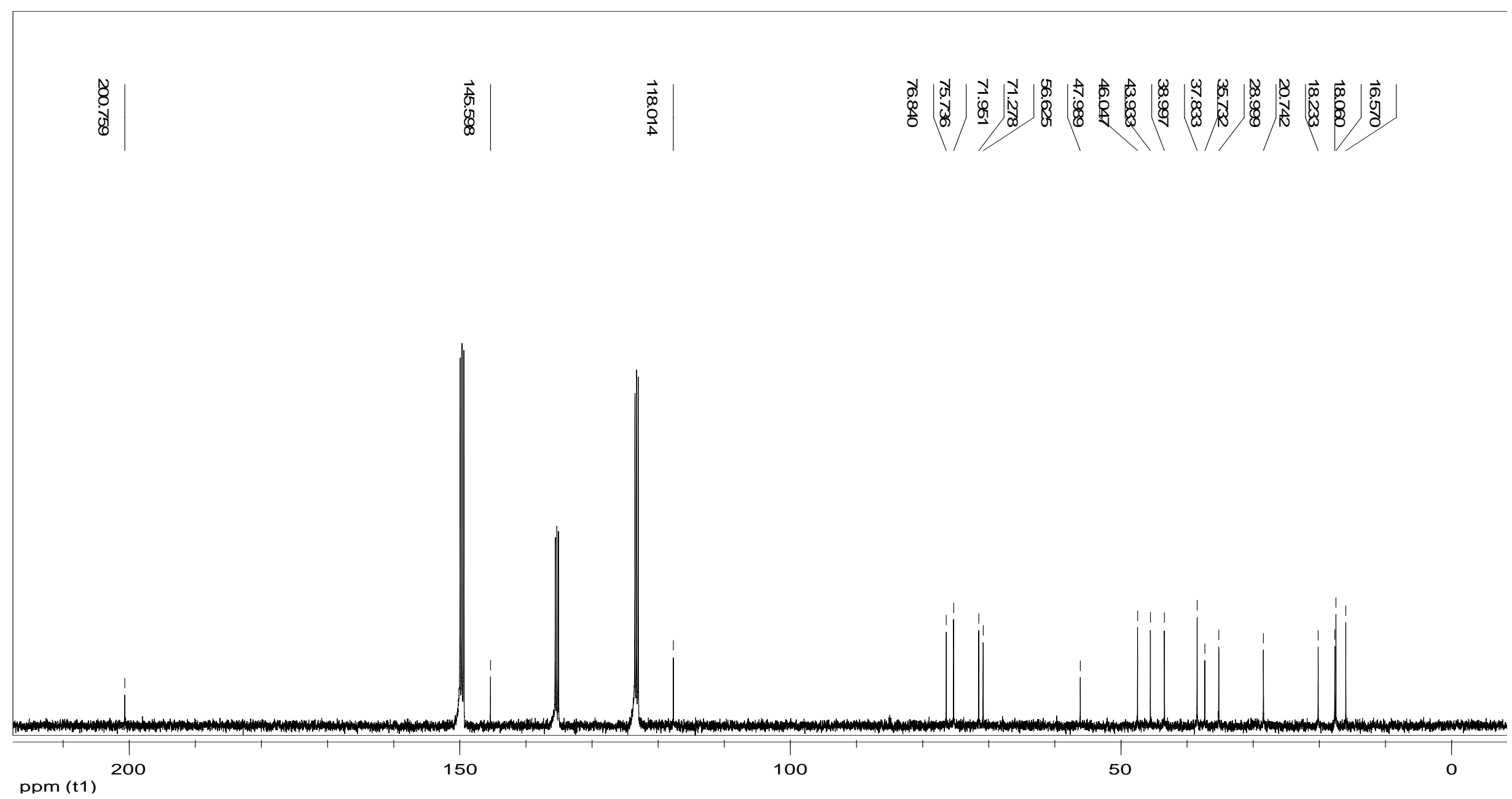


DEPT NMR spectra of alboatisin A (1)
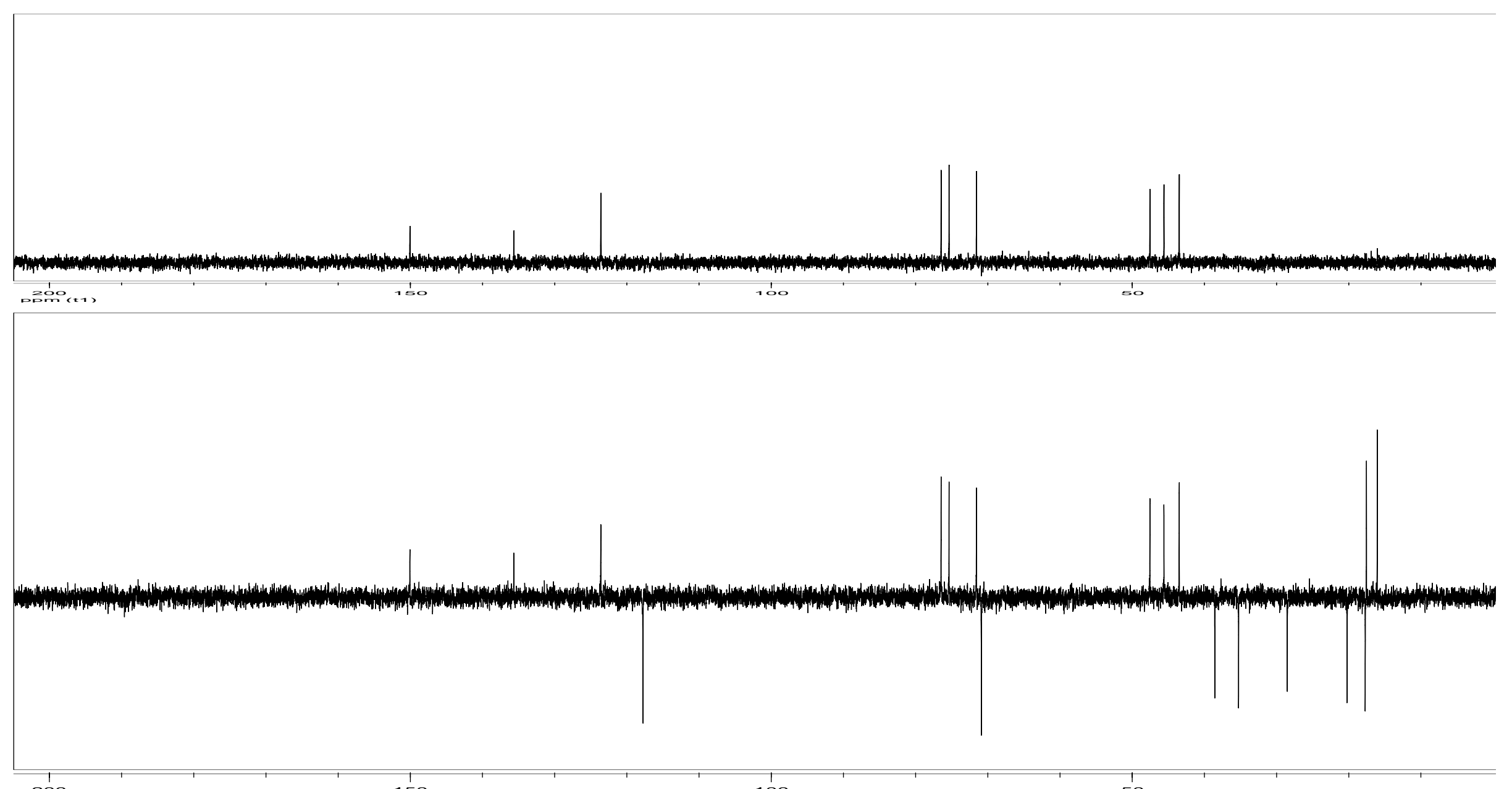

ppm (t1)

150

50 
HSQC NMR spectrum of alboatisin A (1)
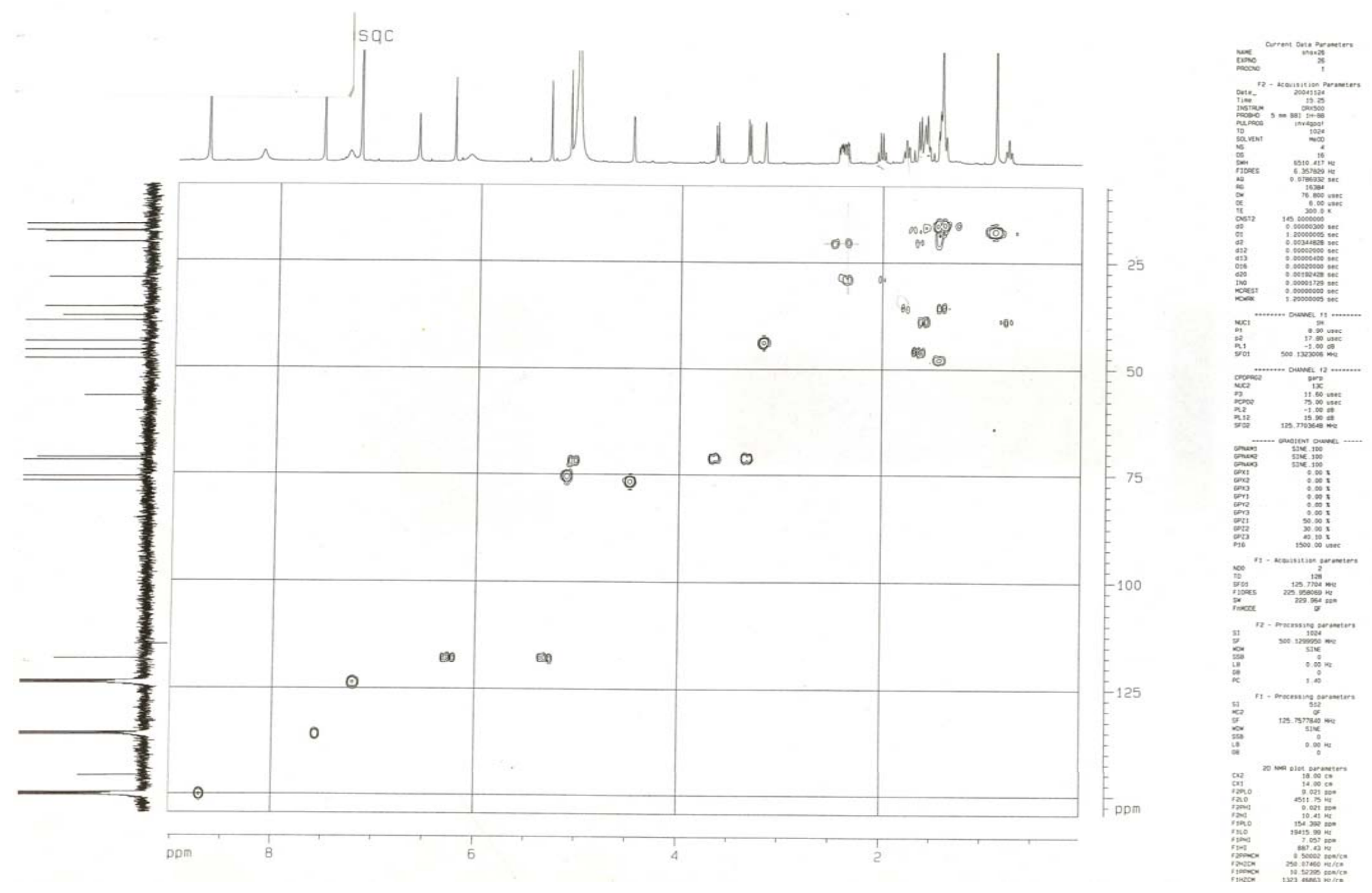


\section{COSY NMR spectrum of alboatisin A (1)}
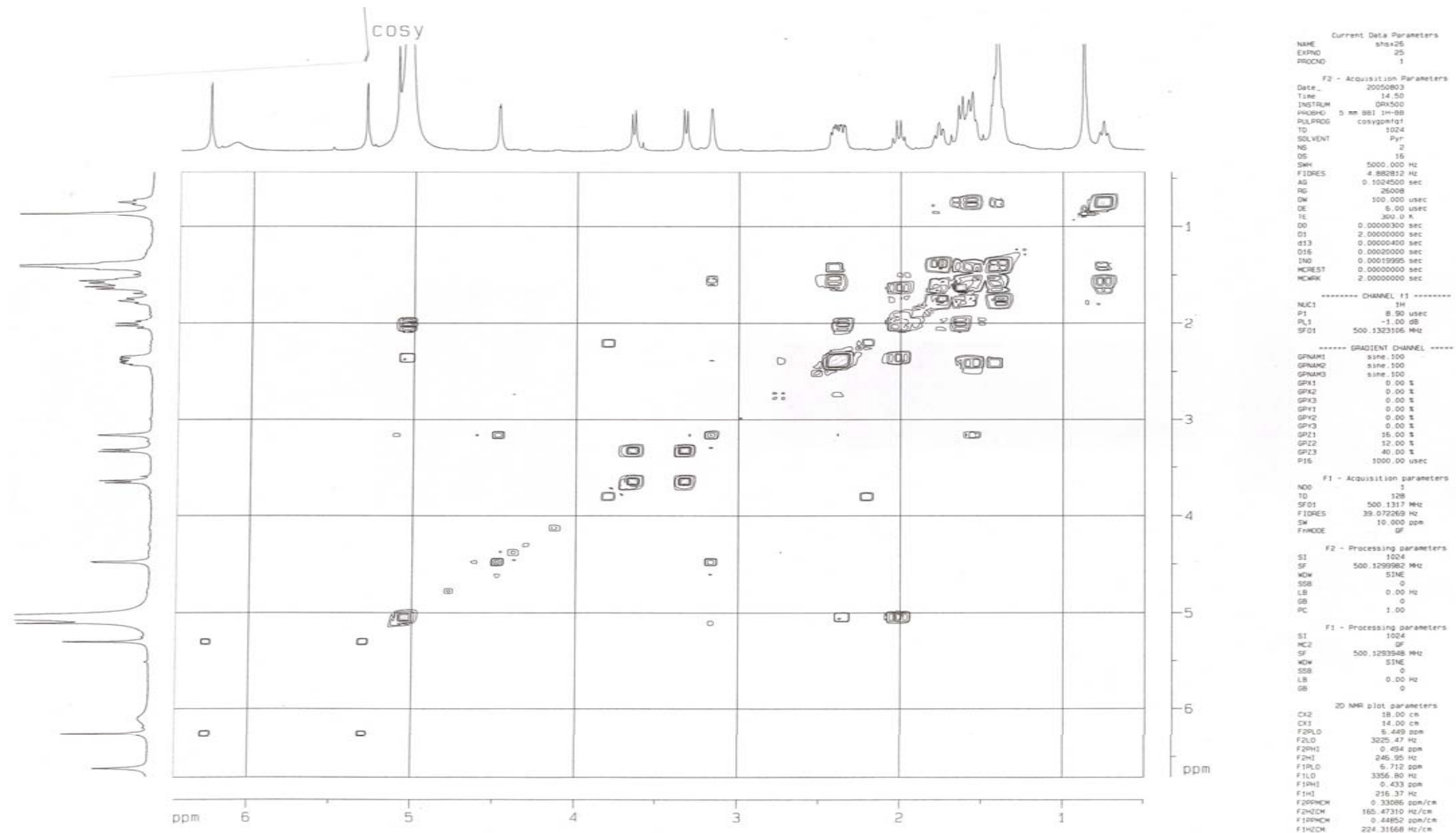
HMBC NMR spectrum of alboatisin A (1)

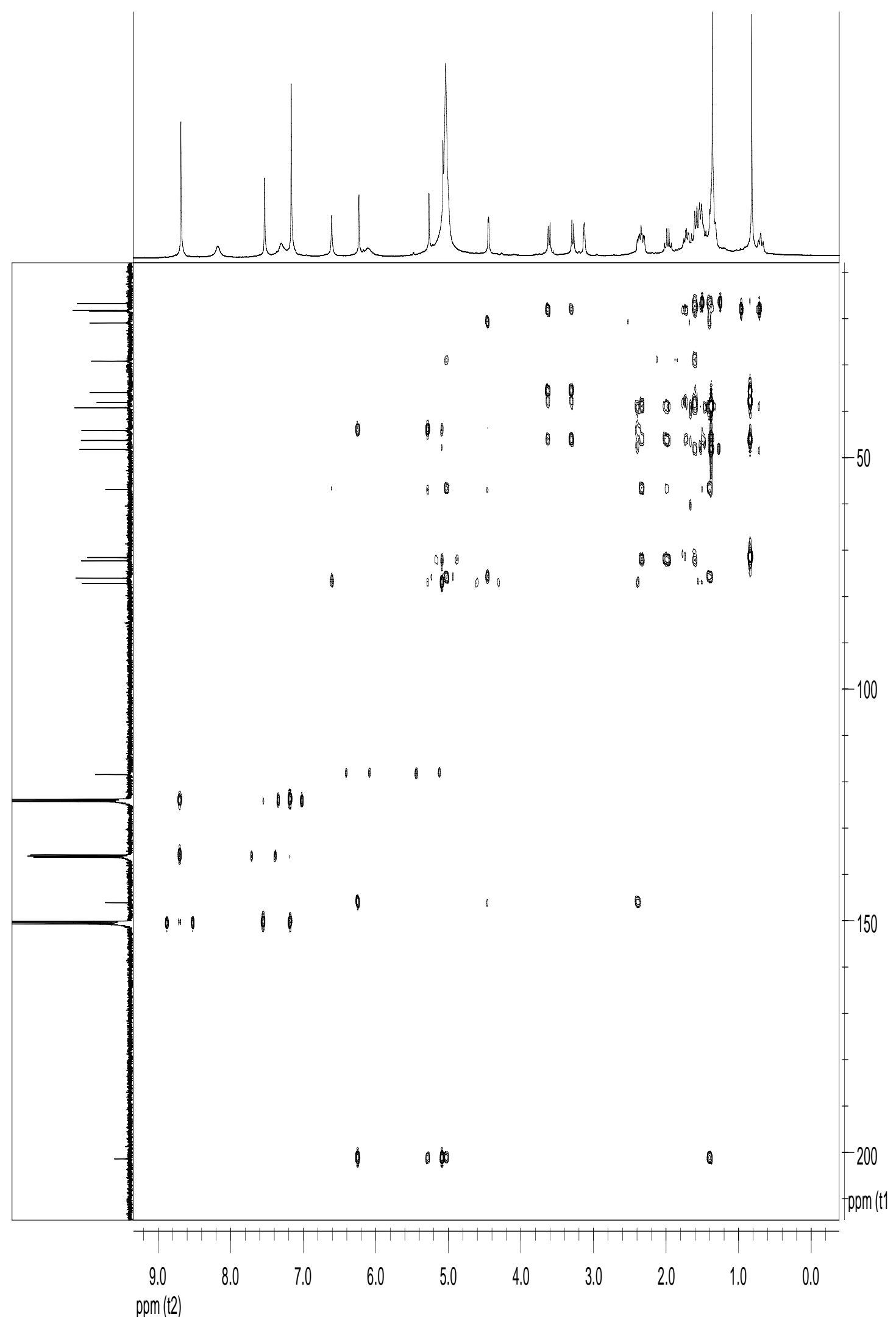


Expanded HMBC spectrum of alboatisin A (1)

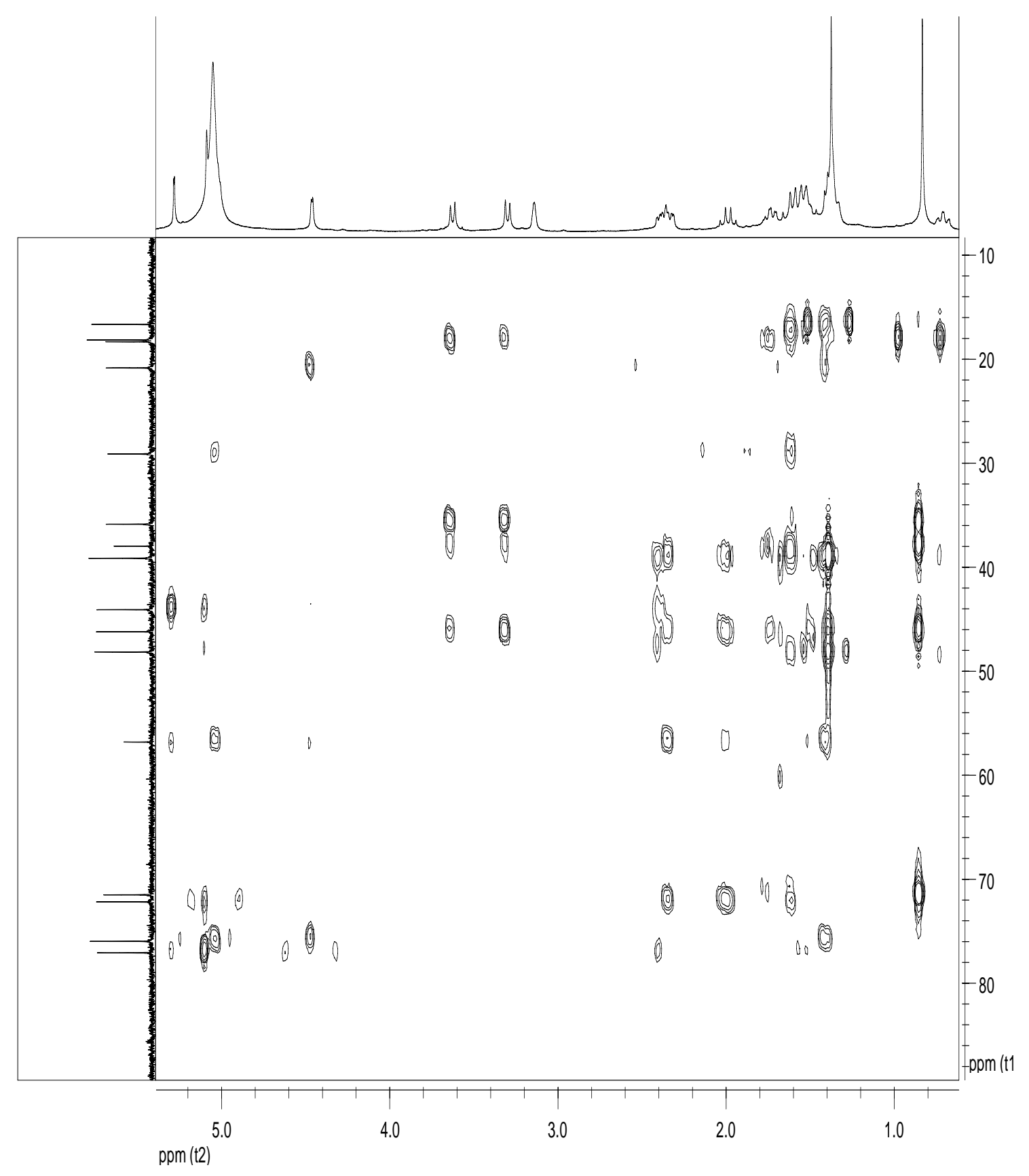


ROESY NMR spectrum of alboatisin A (1)

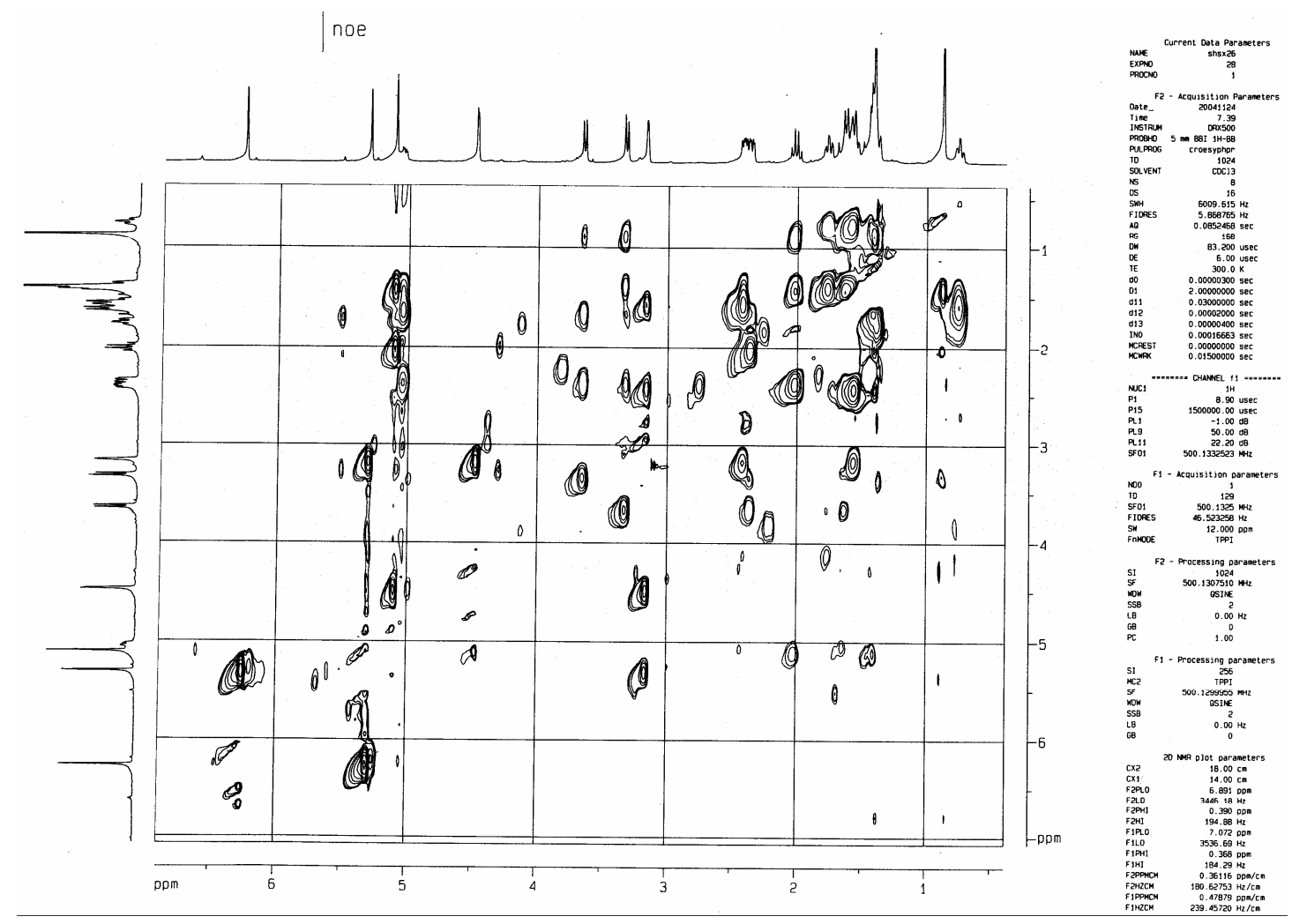




\section{${ }^{1} \mathrm{H}$ NMR spectrum of alboatisin B (2)}

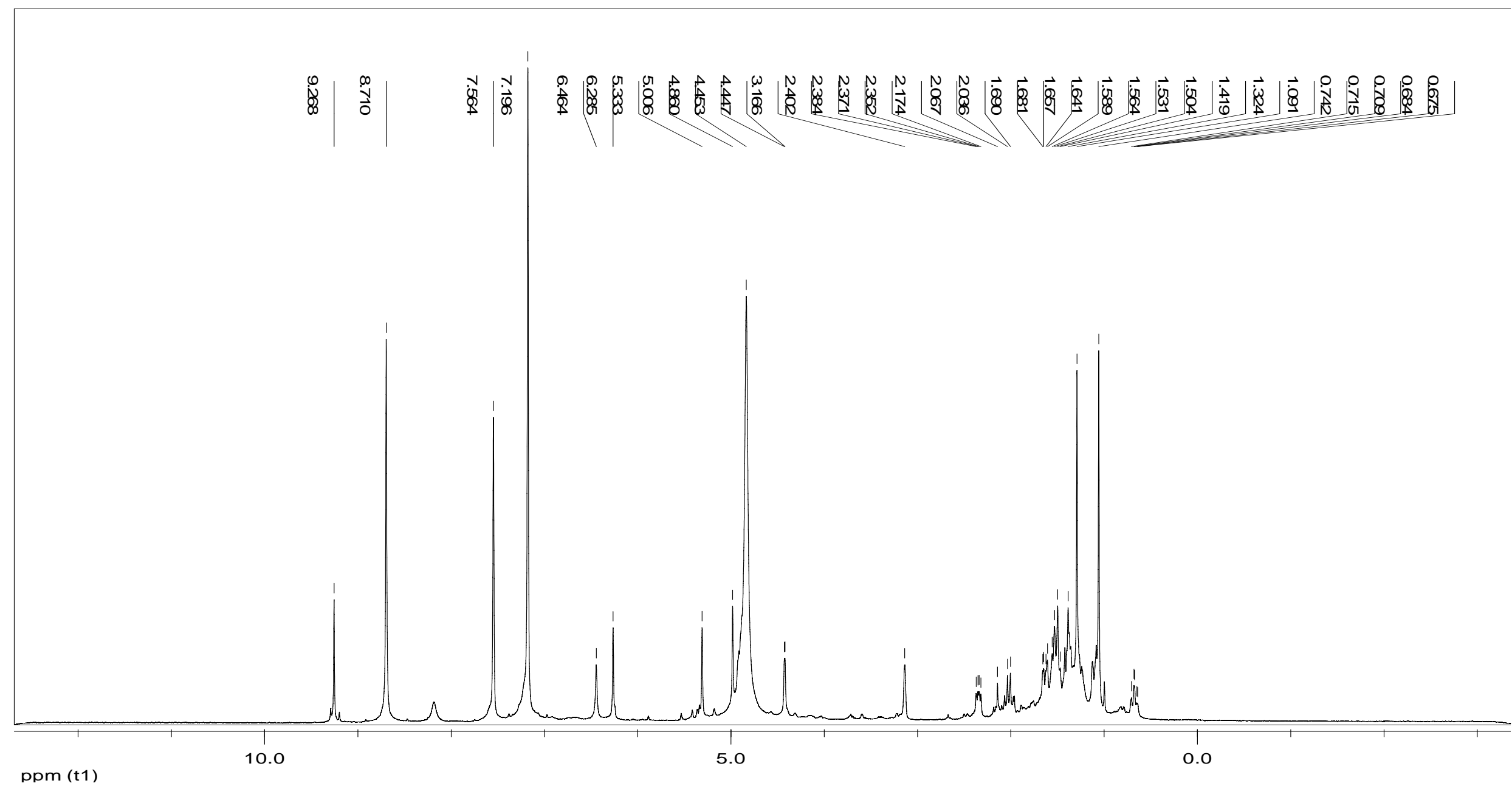


${ }^{13} \mathrm{C}$ NMR spectrum of alboatisin B (2)

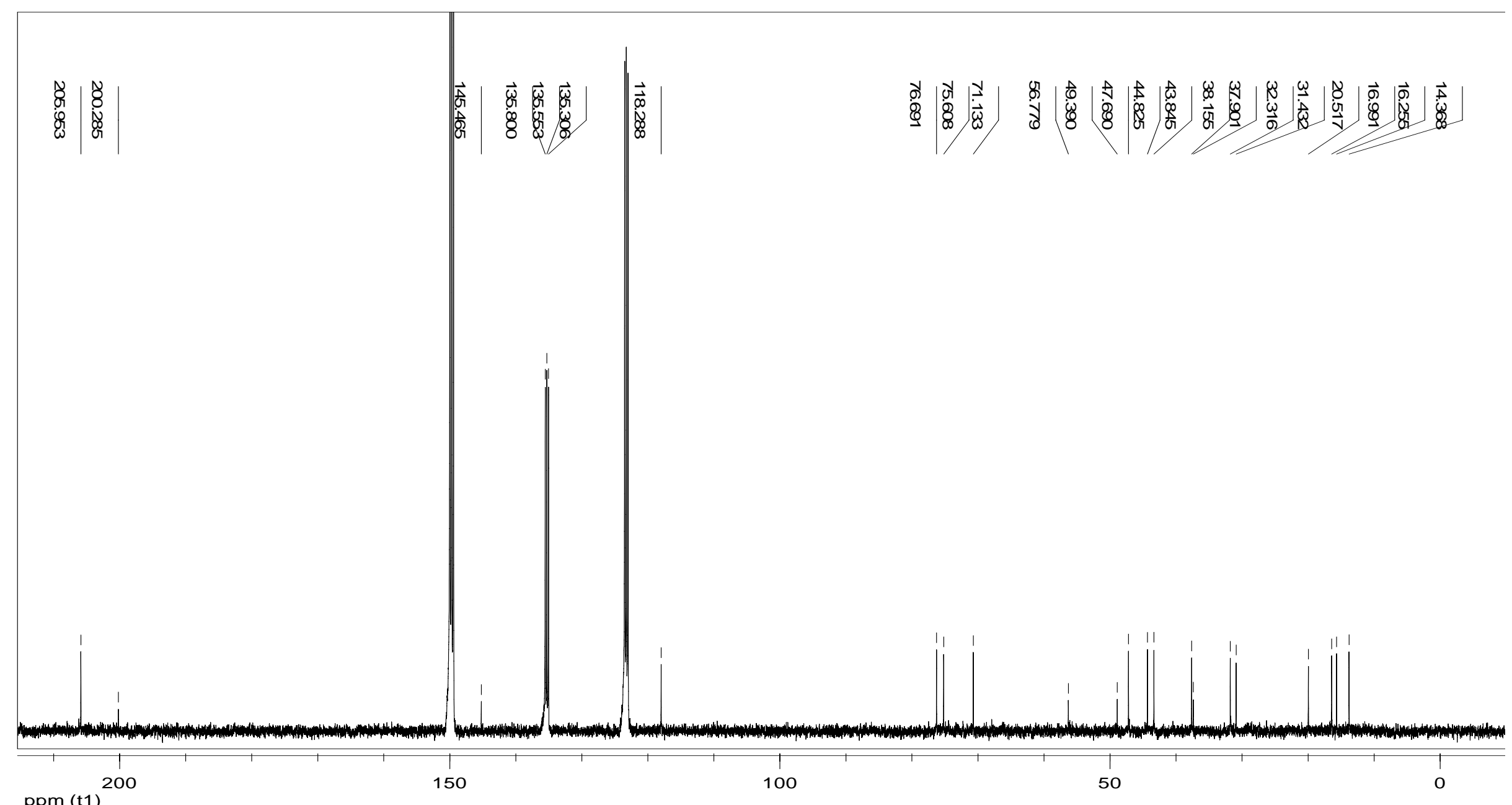

ppm (t1) 
HMBC NMR spectrum of alboatisin B (2)

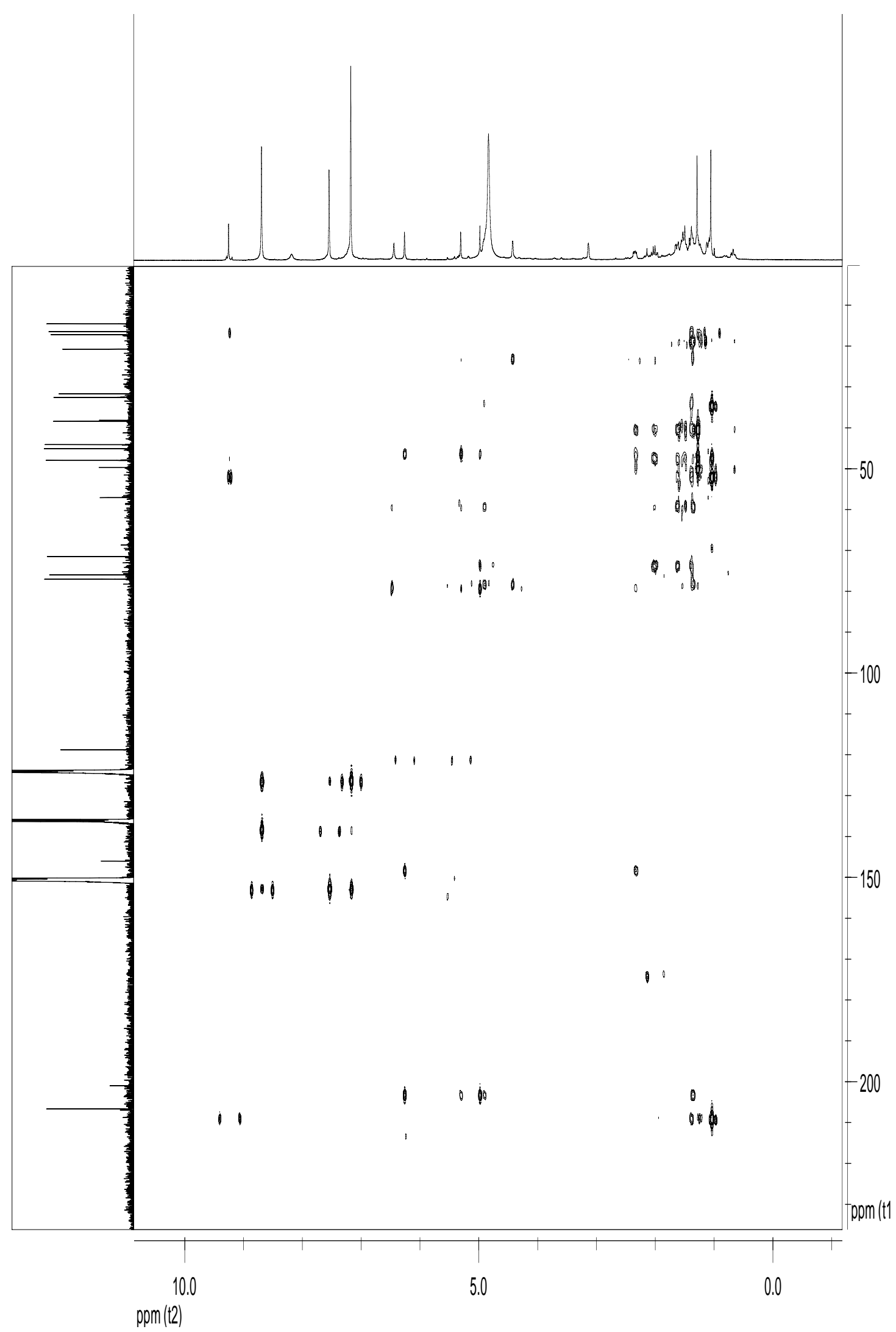


${ }^{1} \mathrm{H}$ NMR spectrum of alboatisin C (3)

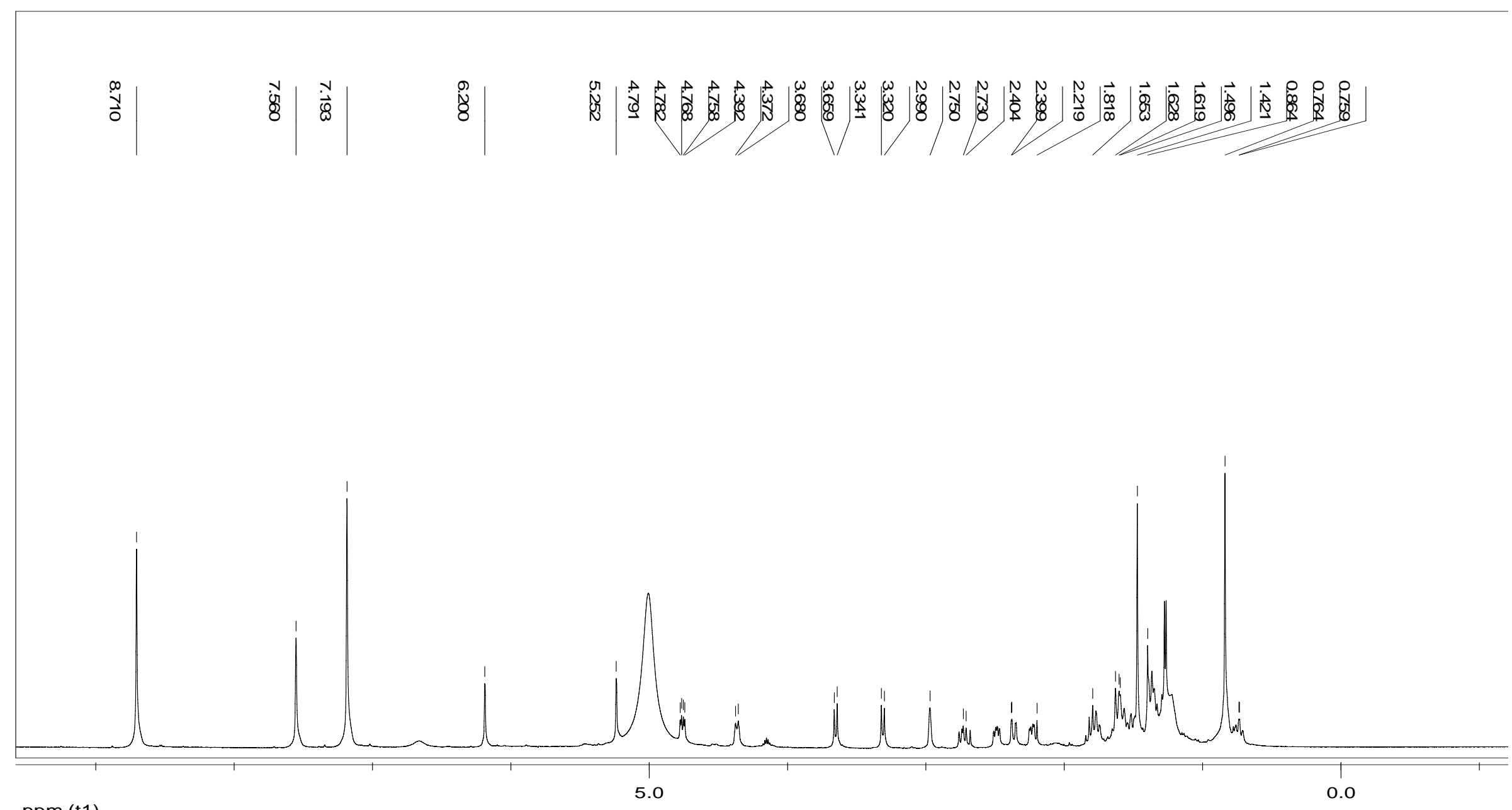


${ }^{13}$ C NMR spectrum of alboatisin C (3)

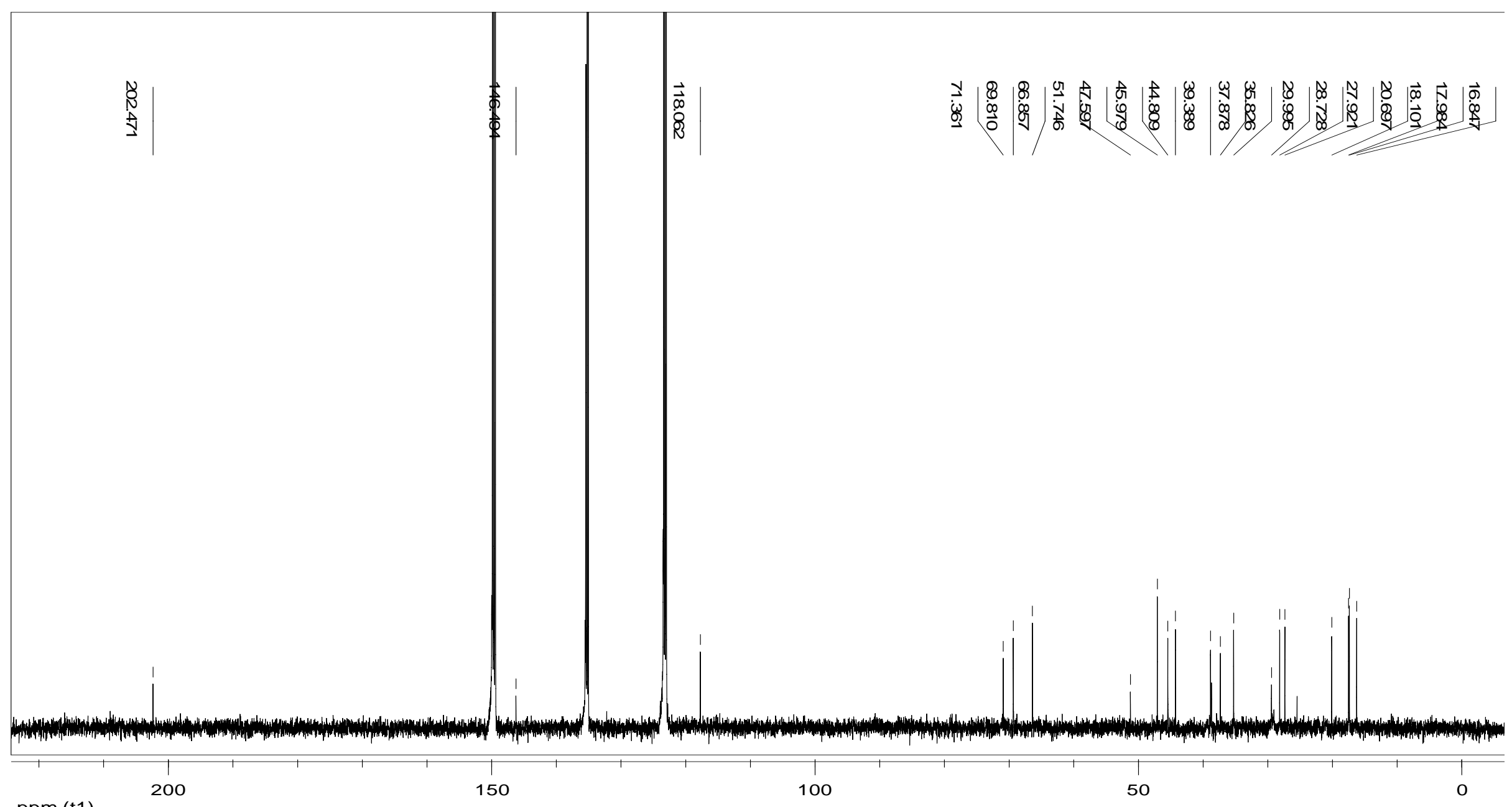

ppm (t1) 


\section{COSY NMR spectrum of alboatisin C (3)}

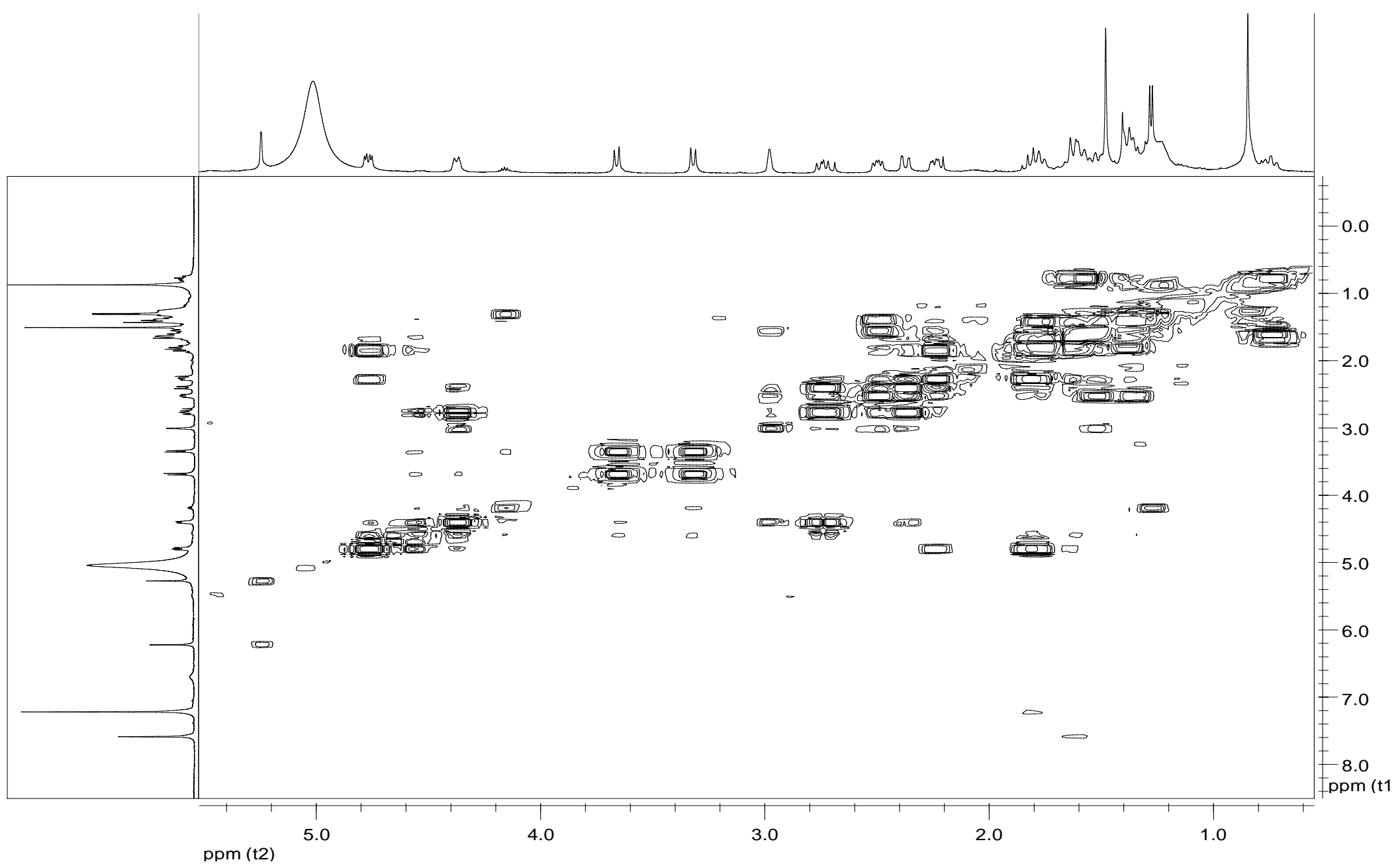

